\title{
Dendritic Cells Activate and Mature after Infection with Mycobacterium tuberculosis
}

\author{
Adane Mihret ${ }^{1,2^{*}}$, Gezahagne Mamo ${ }^{1,3}$, Mesfin Tafesse ${ }^{1}$, Asrat Hailu ${ }^{2}$ and Shreemanta Parida ${ }^{1,4}$
}

\begin{abstract}
Background: Dendritic cells (DCs) can take up an array of different antigens, including microorganisms which they can process and present more effectively than any other antigen presenting cell. However, whether the interaction between the human DC and Mycobacterium tuberculosis represents a defense mechanism by the invaded host, or helping the invader to evade the defense mechanism of the host is still not clearly understood.

Findings: To analyze the interactions between M. tuberculosis and immune cells, human peripheral blood monocyte-derived immature DCs were infected with M. tuberculosis H37Rv wild type strain and flow cytometry was used to analyse cell surface expression markers. The ability of the M. tuberculosis infected DC to induce T cell proliferation using 5 and 6-carboxyfluorescein diacetate succinimidyl ester (CFSE) dilution technique was also investigated. DCs were found to internalize the mycobacteria and show dose dependent infection and necrosis with different multiplicity of infection. Flow cytometry analysis of cell surface expression markers CD40, CD54, CD80, CD83, CD86 and HLA DR in infected DC revealed significant $(p<0.05)$ up regulation following infection with M. tuberculosis in comparison to immature DC with no stimulation. Lipopolysaccharide (LPS) from Salmonella abortus equi, a known DC maturation agent, was used as a positive control and showed a comparable up regulation of cell surface markers as observed with $M$. tuberculosis infected DC. It was revealed that the $M$. tuberculosis infected DC induced T cell proliferation.
\end{abstract}

Conclusion: These data clearly demonstrate that M. tuberculosis induces activation and maturation of human monocyte-derived immature DC as well as induces T cell proliferation in vitro.

Keywords: Dendritic cells, Mycobacterium tuberculosis, T cells, Activation, Flowctometry, CFSE, Proliferation

\section{Background}

Mycobacterium tuberculosis is one of the most ubiquitous and extraordinarily effective human pathogens, with one-third of the global population being infected. Worldwide, an estimated 8.8 million new cases of tuberculosis occurred annually, of which 3.9 million are smear positive [1]. Although effective therapy is now available through the WHO DOTS (Directly Observed Therapy, short course) program and the majority of the world's population is BCG vaccinated, the incidence of TB is still staggering. Clearly, a more effective vaccine against this disease is needed. A greater understanding of the immune response to the organism is necessary in order to develop a vaccine that will confer complete

\footnotetext{
* Correspondence: adane_mihret@yahoo.com

${ }^{1}$ Armauer Hansen Research Institute (AHRI), Addis Ababa, Ethiopia
}

Full list of author information is available at the end of the article protection from infection and, hopefully, from reactivation of a pre-existing infection. In particular, the role of Dendritic cells (DCs) in the initiation of specific T cell immunity to $M$. tuberculosis has not been clearly elucidated.

DCs comprise a family of antigen presenting cells that act like 'conductors' of the immune response in their capacity to orchestrate signals derived from the different parts of the immune system [2]. DCs show a unique functional duality during their development, designed to ultimately provide secondary lymphoid tissues with useful information about the antigenic composition in the periphery. At the immature stages of development, DCs resident in peripheral tissues are specialized in antigen capture, acting as sentinel cells (high intracellular MHC II, endocytosis, phagocytosis, high CCR1, CCR5, and CCR6; low CCR7 and low CD40, CD54, CD80, CD83, CD86 and CD58).

\section{C)


After antigen uptake, DC rapidly migrate to the draining secondary lymphoid organ. During this migration, DC undergo a maturation process which is characterized by down-regulation of the capacity to capture antigen and up regulation of antigen processing and presentation, expression of costimulatory molecules and of dendritic morphology (high surface MHC II, low endocytosis, low phagocytosis, low CCR1, CCR5, CCR6, high CCR7 and high CD40, CD54, CD80, CD83, CD86 and CD58) [3].

Whether the encounter between the human dendritic cells and $M$. tuberculosis represents a defence mechanism by the invaded host, or helping the invader to evade the defence mechanism of the host is still not clearly understood. Henderson et al reported that human monocyte derived DC phagocytose $M$. tuberculosis efficiently, suggesting a role for this important cell in the early response to $\mathrm{TB}$ infection. Infection with this pathogen resulted in up regulation of MHC I and MHC II, CD40, CD54, CD58, and CD80 [4], a phenotype consistent with the activation of the $\mathrm{DC}$, suggesting that infected DC produce cytokines that lead to maturation, and possibly to migration and antigen processing and presentation. In contrast to these findings, other workers have reported that $M$. tuberculosis inhibits maturation of human monocyte-derived dendritic cells in vitro and infected DCs show some up regulation of human monocyte-derived DC surface expression of maturation markers such as CD25, CD83, CD 40, CD80, CD86 and the antigen presenting molecules $\mathrm{MHC}$ I and MHC II. This was minimal compared with that induced by the maturation cocktail of TNF $\alpha$, IL-1 $\beta$, and $\mathrm{PGE}_{2} . M$. tuberculosis infected human monocyte-derived DC are compromised in their ability to activate naive $\mathrm{T}$ cells and were poor inducers of autologous $\mathrm{T}$ cell proliferation and cytokine production [5]. However, in this study our data demonstrate that immature human DC can take up M. tuberculosis bacilli in vitro and the infection induces DC activation, as measured by up regulation of cell surface markers accompanied by $\mathrm{T}$ cell proliferation.

\section{Materials and methods}

\section{Media and Reagents}

The medium used throughout the study was RPMI 1640 with L-glutamine (Sigma), supplemented with 1\% penicillin streptomycin (Difco) and 10\% FCS (Sigma), (RPMI-FCS). Human rGM-CSF and rIL 4 were purchased from Cytolab. LPS from Salmonella abortus equi was purchased from Sigma, and CFSE was purchased from Molecular Probes.

Monoclonal antibodies conjugated with fluorochromes against CD40 FITC (5C3 clone and mouse IgG $1, k$ antibody composition), CD54 PE (HA58 clone and mouse $\mathrm{IgG}_{1, \mathrm{k}}$ antibody composition), CD80 PE (L307.4 clone and mouse IgG $_{1, k}$ antibody composition), CD83 FITC (HB15e clone and Mouse IgG $_{1, k}$ antibody composition), CD86 PE (FUN-1 clone and mouse IgG $_{1}$, k antibody composition), HLA DR FITC (G46-6 clone and mouse $\mathrm{IgG}_{2 \mathrm{a}, \mathrm{k}}$ antibody composition), CD14 FITC (M5E clone and mouse $\operatorname{IgG}_{2 \mathrm{a}, \mathrm{k}}$ antibody composition), CD3 PE (UCHT1 clone and mouse IgG I $_{1}$ antibody composition), FITC conjugated mouse IgG1 Isotype control (MOPC-21 clone and mouse $\operatorname{IgG}_{1, k}$ antibody Isotype), PE conjugated mouse IgG1, ${ }_{\mathrm{k}}$ Isotype control (MOPC-21 21 clone and mouse $\operatorname{IgG}_{1, k}$ antibody isotype), FITC conjugated Mouse IgG 2a,k (G155-178 clone and mouse $\operatorname{IgG}_{2 \mathrm{a}, \mathrm{k}}$ antibody isotype and 7AAD were purchased from BD Pharmingen. CD14 microbead conjugated antibodies and columns were purchased from Miltenyi Biotech.

\section{Laboratory Methods \\ Generation of immature human Dendritic Cells in vitro}

Immature DCs were generated from positively selected CD $14^{+}$cells as described previously [6] with minor modifications. In brief, monocytes were purified by positive selection of $\mathrm{CD} 14^{+}$cells from Peripheral Blood Mononuclear Cells (PBMCs) of apparently healthy Ethiopian male and female subjects by using a magnetic cell separator (VarioMacs, Miltenyi Biotech, Germany) according to the manufacturer's instruction. CD $14^{+}$cells were then incubated in six-well tissue culture plates (at a concentration of approximately $2 \times 10^{6} /$ well in a volume of $3 \mathrm{ml}$ ) for 7 days at $37^{\circ} \mathrm{C}$ in an atmosphere of $5 \% \mathrm{CO}_{2}$ in complete medium (RPMI 1640 with L-glutamine supplemented with $1 \%$ penicillin streptomycin and 10\% FBS), $50 \mu \mathrm{M}$ 2-mercaptoethanol, $50 \mathrm{ng} / \mathrm{ml}$ human recombinant GM-CSF and $50 \mathrm{ng} / \mathrm{ml}$ human recombinant IL-4. After 7 days of incubation, the non-adherent cells (Immature DCs) were harvested and placed in 24well plates containing $2 \mathrm{ml}$ complete medium without antibiotics. CD14- cells were stored frozen in liquid nitrogen and used as autologus $\mathrm{T}$ cells to do mixed leukocyte reaction.

\section{Mycobacteria preparation}

H37Rv was grown in Middlebrook 7H10 agar (Difco Laboratories, Detroit, MI) at $37^{\circ} \mathrm{C}$ under a humidified $5 \%$ CO2 atmosphere for 2 weeks. Bacterial suspensions were prepared by dispersing colonies with glass beads in RPMI 1640. The tubes were vortexed and allowed to stand for $30 \mathrm{~min}$ to let larger particles settle. The upper supernatant was stored at $-80^{\circ} \mathrm{C}$ until use. Colony-forming units were counted by the standard viable count technique in Middlebrook 7H10 agar plates.

\section{Infection of DC with M. tuberculosis}

Immature DC were plated in 24-well plates at $2 \times 10^{5}$ cells in $2 \mathrm{ml}$ of complete media per well without antibiotics and then were infected with H37Rv at a 
multiplicity of infection (MOI) of 5 in the Biological Safety Level-3 (BSL-3) lab. After 6 hours of incubation at $37^{\circ} \mathrm{C}$ in $5 \% \mathrm{CO}_{2}$ incubator, infected cells were washed three times with complete medium without antibiotics at low speed $(600 \mathrm{rpm})$ to remove extracellular bacteria and were incubated in fresh medium for a further 2 days. Infected DC were incubated in $0.02 \%$ EDTA for 15 minutes at RT before harvesting to obtain the total cellular content of each well. This was followed by a single wash with complete media without antibiotics. The percentages of infected and necrotic cells were estimated in each experiment by staining aliquots of cells by ZiehlNeelsen method for acid-fast bacteria and 7-amino-actinomycin D (7-AAD) respectively. In addition, infected DCs were evaluated after 48 hours for both cell surface phenotype and extent of autologous $\mathrm{T}$ cell stimulation using flow cytometry as described below.

\section{Flow Cytometric Analysis of Surface Markers}

All groups of DC (Immature DC alone, Immature DC infected with H37Rv M. tuberculosis strain, and stimulated with $1 \mu \mathrm{g} / \mathrm{ml}$ LPS for 48 hours) surface marker expression was assessed by flow cytometry (FACScan, $\mathrm{BD})$ using direct double immunofluorescence labelling technique (CD40 FITC/CD80 PE, HLA DR FITC/CD54 PE, CD83 FITC/CD86 PE). In brief, $2 \times 10^{5}$ cells were aliquoted into tubes and washed once with FACS medium (PBS, $1 \%$ FBS and $0.1 \%$ Sodium azide) at $400 \mathrm{~g}$ at $4{ }^{\circ} \mathrm{C}$ for 10 minutes and incubated with $15 \mu \mathrm{l}$ fluorochrome conjugated monoclonal antibodies of each surface antigen for 30 minutes at $4{ }^{\circ} \mathrm{C}$. Then, cells were washed an additional three times with FACS medium. Dead cells were eliminated from the analysis by staining with 7-amino-actinomycin D (7AAD). The values were reported as Mean Fluorescence Intensity (MFI) of the total events counted $(\mathrm{n}=10,000)$. DCs necrosis was assessed by 7-AAD Staining Assay. In all experiments evaluating DC phenotypic maturation, dead cells (7AAD bright) were excluded from analysis.

\section{Cytokine assay}

At the end of the incubation period, culture supernatants of DC were collected, filtered with $0.2 \mu \mathrm{m}$ and stored at $-80^{\circ} \mathrm{C}$. The levels of TNF $\alpha$, IL-12 and IL-10 were determined by enzyme-linked immunosorbent assay (ELISA) using recombinant cytokines for generating standard curves. Anti-human TNF $\alpha$, IL-12 and IL-10 mAb, biotin mouse anti-human TNF $\alpha$ IL 12 and IL-10 mAb and standard were purchased from Pharmingen.

\section{Mixed Leukocyte Reaction}

The ability of M. tuberculosis infected DC to stimulate T cells was assessed using a mixed DC-autologous-T-cell reaction. Infected DCs were used to stimulate autologous CD 14- PBMC (purified by magnetic beads following CD $14^{+}$positive selection). A total of $2 \times 10^{5}$ CFSE stained CD 14- PBMC (responder cells) were added to each well in $100 \mu \mathrm{l}$ complete media on U bottom, 96 well plates (BD, Pharmingen). DCs (stimulator cells) were harvested following stimulation with medium alone/LPS/M. tuberculosis, washed twice, resuspended in complete medium and then added to the responder cells at a ratio of $1: 10$ and 1:100. After 5 days culture in 5\% $\mathrm{CO}_{2}$ at $37^{\circ} \mathrm{C}$, cells were harvested and $\mathrm{CD} 3$ positive cells were analysed for their CFSE staining intensity.

The number of cells that had proliferated was determined by gating on the lineage positive CFSE ${ }^{\mathrm{dim}}$ subset. The cell division index (CDI) was calculated based on a fixed number (usually 5,000) of $\mathrm{CFSE}^{\text {bright }} \mathrm{CD}^{+}$cells, with the following formula:

$$
\text { CDI } \frac{\text { Number of } \mathrm{CD}^{+} \mathrm{CFSE}^{\mathrm{dim}} \text { cells with antigen }}{\text { Number of } \mathrm{CD}^{+} \mathrm{CFSE}^{\mathrm{dim}} \text { cells without antigen }}
$$

A CDI of greater than 2 was considered to represent a positive response. A CDI of 2.0 means that there are twice as many divided cells with antigen than without [7].

\section{Ethical Consideration}

The project obtained ethical clearance from the Medical Faculty Research and Publication committee of the Addis Ababa University, AHRI/ALERT Ethical Review Committee and the National Ethical Review Committee before commencement of the study.

\section{Data Analysis}

The mean fluorescence intensity data were analysed using a non-parametric Wilcoxon signed rank test, and results were considered significant if $p \leq 0.05$ and for the proliferation test CDI values greater than 2 was taken as a positive result [7]

\section{Results}

Viability of Dendritic Cells Following Infection with H37Rv Immature DCs were infected with H37Rv at different multiplicity of infection (MOI). Twelve experiments from different subjects were done to determine the optimum MOI to be used in subsequent experiments. The percentage of the cells infected at the various MOIs at the time of collection varied from 26 to $89 \%$ (data not shown). Forty-eight hours after infection, cells were collected and viability was determined by staining with 7 AAD. Infected cells were stained with Zihel-Nielsen for the presence of intracellular acid-fast bacteria. At the lowest MOI of 3 , only $31.6 \pm 2.6 \%$ of the cells stained positive for intracellular bacteria and $5.6 \%$ were positive for 7-AAD. At MOI of 5, the percentage of cells staining positive for intracellular bacteria increased to $60 \pm 1.3 \%$ 
and the percentage of cells positive for 7-AAD was 11.1 $\pm 4.5 \%$, whereas at an MOI of $10,84.4 \pm 4.5 \%$ of cells stained positive for intracellular bacteria. At the highest MOI of 10, although the percentage of cells positive for intracellular bacteria was higher, a significant proportion (18.2\%) of the DC were not viable. The mean percentage of viable immature DC was $97.7 \%(96.8-98.6 \%)$ in the case of uninfected cells. On the basis of these data, a MOI of 5 was chosen as optimal for further experiments.

\section{Maturation of Dendritic Cells}

Functional activities of DC were determined by surface expression of adhesion and co-stimulatory molecules. To investigate the effect of $M$. tuberculosis H37Rv strain infection on the DC phenotype, the expression of the intracellular adhesion molecule (ICAM 1 and CD54), co-stimulatory surface molecules (B7-1 (CD80), B7-2 (CD86) and CD40) and MHC II by immature, H37Rv infected and LPS stimulated DC were assessed using 18 experiments from different subjects by flow cytometry. The cells were infected with H37Rv at a multiplicity of infection of 5 or stimulated with LPS at a concentration of $1 \mu \mathrm{g} / \mathrm{ml}$ or immature DC treated with media alone and analyzed $48 \mathrm{hr}$ post treatment for the expression of the above-mentioned surface molecules. Significant $(\mathrm{p}<$ $0.05)$ up regulation in cell surface expression of CD40, CD80, CD54 and MHC class II and CD86 and CD83 (Table 1) were observed both in LPS stimulated and $\mathrm{H} 37 \mathrm{Rv}$ infected in comparison to negative control DC with media alone without any stimulation/infection.

\section{Cytokine ELISA}

The production of TNF $\alpha$ and IL 12 was found to be significantly higher in all infected MoDC compared to control (Figure 1). IL10 was also measured in the MoDC culture supernatants but their levels were not statistically significant compared to unstimulated groups $(\mathrm{P}>0.05)$.

Table 1 Expression of various phenotypic markers on dendritic cells before and after infection

\begin{tabular}{llll}
\hline $\begin{array}{l}\text { Phenotypic } \\
\text { markers }\end{array}$ & $\begin{array}{l}\text { Control } \\
\text { (Uninfected) }\end{array}$ & $\begin{array}{l}\text { LPS } \\
\text { stimulated }\end{array}$ & $\begin{array}{l}\text { H37Rv } \\
\text { infected }\end{array}$ \\
\hline CD 40 & $40.7 \pm 16.4$ & $153.3^{*} \pm 15.8$ & $82.8^{*} \pm 12.8$ \\
CD 80 & $39.2 \pm 6.7$ & $109.5^{*} \pm 8.3$ & $92.4^{*} \pm 6.9$ \\
HLA DR & $153.4 \pm 18.6$ & $639.9^{*} \pm 12.7$ & $480^{*} \pm 12.4$ \\
CD 54 & $102.3 \pm 16.3$ & $292.2^{*} \pm 13.4$ & $218.7^{*} \pm 11.4$ \\
CD 83 & $3.2 \pm 1.1$ & $11.4^{*} \pm 2.4$ & $8.1^{*} \pm 1.5$ \\
CD 86 & $22.4 \pm 3.2$ & $86.2^{*} \pm 6.2$ & $78.4^{*} \pm 5.7$ \\
\hline
\end{tabular}

Expression of each marker is presented as percentage positive cells. The values represent mean \pm SEM of at least 17 independent experiments for each condition. The statistical significance is shown as compared to uninfected; ${ }^{*} \mathrm{P}<0.05$

\section{Mixed Leukocyte Reaction}

The ability of $M$. tuberculosis infected DC to stimulate autologous $\mathrm{T}$ cells was assessed using mixed DC-autologous $\mathrm{T}$ cell reaction. The $\mathrm{T}$ cell proliferation was quantified by CFSE dilution techniques described in the methodology part. The results were expressed as the mean Cell Division Index (CDI) from each experiment.

The DC stimulated with LPS and infected with H37Rv augments $\mathrm{T}$ cells proliferation both at 1:10 (Mean CDI $=34.2$ and 15) and 1:100 (Mean CDI = 15.6 and 10.6) DC: $\mathrm{T}$ cell ratio respectively (Figure 2 ).

\section{Discussion}

The DC activation process that results in a mature phenotype appears to be a crucial step in generating a specific immune response. We used the virulent $M$. tuberculosis $\mathrm{H} 37 \mathrm{Rv}$ strain to infect DC. The percentage of infected DC was proportional to the MOI. In this study, we used a MOI of 5, which resulted in $60 \pm 1.3 \%$ level of infection. Higher MOI of 12.5 caused a large amount of necrosis [8]. In our study we have found $5.6 \%, 11.1 \%$ and $18.2 \%$ of cell death at a MOI of 3,5 and 10 respectively, which clearly shows at higher MOI the more cell death will be.

This study demonstrates that the exposure of monocyte-derived immature DC to H37Rv M. tuberculosis strain leads to activation or maturation of DC. Significant increases in cell surface expression of accessory molecules, CD40, CD80, CD86 and CD83, CD54), and MHC class II maturation marker $(\mathrm{p}<0.05)$ were observed. The increase in the expression of CD40, CD80 and CD86 may result in an increased capacity of DC to trigger proliferative responses and IFN- $\gamma$ production by $\mathrm{T}$ cells against $M$. tuberculosis infection. In addition, CD40-CD40L interaction is the most potent stimulus in up regulating the expression of CD54, CD80, and CD86 molecules on DC [9].

The increment of expression of CD54 would help to increase the strength of adhesion between $\mathrm{T}$ cells and $\mathrm{DC}$, thus allowing the TCR to be engaged by antigen to transduce the necessary signals, since it is known that the affinity of TCR for peptide MHC complex is quite low and the off rate of this interaction is also rapid.

Although the precise function of CD83 is still unknown, several pathogens such as HSV-1 [10] and Measles virus [11] have been shown to interfere with CD83-expression in infected DC, which then consequently also interfered with DC-mediated T-cell stimulation. Furthermore, in experiments where the CD83 mRNA transport from the nucleus into the cytoplasm was specifically inhibited, and thus CD83 expression was blocked, the T-cell stimulatory capacity of these DC was also inhibited [12]. All these reports clearly suggested an important role for CD83 during the induction of 


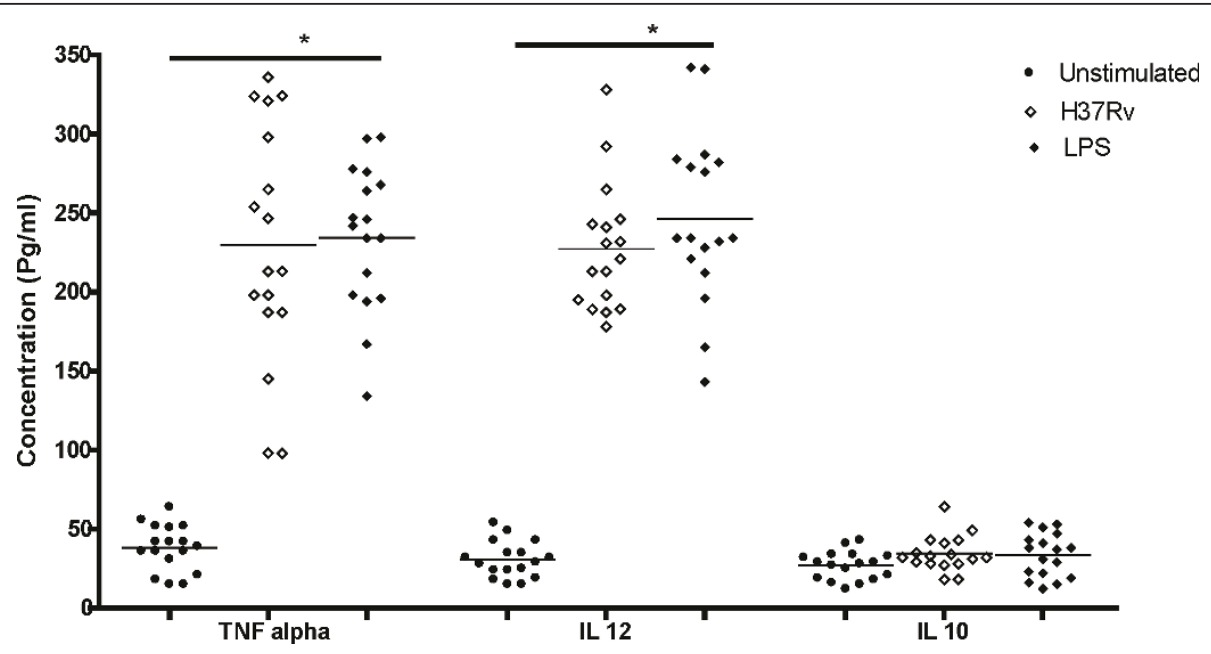

Figure 1 Cytokine Secretion by MoDCs infected with M. tuberculosis and stimulated with LPS. The production of TNF $\alpha$ and IL-12 was found to be significantly higher $(P<0.05)$ in all H37Rv infected and LPS stimulated MoDC compared to control whereas the production of IL10 was not statistically significant $(P>0.05)$.

immune responses. The up regulation of HLA DR molecules on the surface of DC may also ensures that T cells will have the chance to recognize and respond to $\mathrm{MHC}$ associated peptides. In general, up regulation of these cell surface markers that are important in antigen presentation and $\mathrm{T}$ cell stimulation indicates that the dendritic cells are maturing and preparing for the presentation of antigen to $\mathrm{T}$ lymphocytes.

Other workers also reported that DC readily internalized M. tuberculosis bacilli and subsequently displayed phenotypic changes including up regulation of various cell surface molecules important in initiating immune responses and downregulation of phagocytic activity, as well as producing inflammatory cytokines [12].

Other workers have also shown that pulmonary DC traffic from the lungs to the draining lymph nodes to present inhaled antigens to $T$ cells after bacterial infection [13]. In response to $M$. tuberculosis infection, DC shift to an antigen presenting phenotype and can stimulate $\mathrm{T}$ cells from the spleens and lungs of infected mice in vitro [14]. M. tuberculosis infected DCs have been observed in vivo in infected mice [15].

In contrast to our findings, other workers have reported that $M$. tuberculosis targets DC-specific C-type lectin intercellular adhesion molecule-3-grabbing nonintegrin (DC-SIGN) both to infect DC and to down-regulate maturation of human monocyte-derived DCs. $M$. tuberculosis induced minimal up regulation of the chemokine receptor CCR7, the co-stimulatory molecules CD40, CD80, and CD86 and the antigen presenting molecules MHC class I and MHC class II [16].

The discrepancy between the previous reports and this study may arise from two points: multiplicity of infection and the positive control that was used. The MOI that they used was 3 with an infection of approximately $25 \%$. This indicates a low proportion of infection and with a possibility of very few bacilli per cell, which consequently could lead to a false negative or low maturation of DC. The other point is that they compared the state of maturation or MFI of $M$. tuberculosis infected DC with DC stimulated with a maturation cocktail and this optimal positive control would give a higher MFI as compared to M. tuberculosis. However, we used a MOI of 5 that would give relatively high number of bacilli per cell and we used a bacterial cell wall component (LPS) as a positive control, which would possibly give us a picture more comparable to natural pathogens.

We also examined the ability of $M$. tuberculosis infected DC to induce antigen specific $\mathrm{T}$ cell proliferation. Maturation does not necessarily mean that the cell is functionally capable of presenting antigen to $\mathrm{T}$ cells. The ability to measure autologous $\mathrm{T}$ cell proliferation in response to antigen was assessed by the CFSE dilution technique. Both at 1:10 and 1:100 DC: T cell ratio, infected DC induced positive $\mathrm{T}$ cell proliferation (CDI > 2 ), although $\mathrm{T}$ cell proliferation was stronger at a 1:10 rather than 1:100 DC: $\mathrm{T}$ cell ratio, which could in fact be due to the higher number of DCs. The ability of $\mathrm{H} 37 \mathrm{Rv}$ infected DCs to induce $\mathrm{T}$ cell proliferation was slightly lower than LPS and this may be due to the higher concentration of LPS that we used to stimulate dendritic cells.

\section{Conclusion}

In this study, we have clearly demonstrated that $M$. tuberculosis infection resulted in a phenotype change 

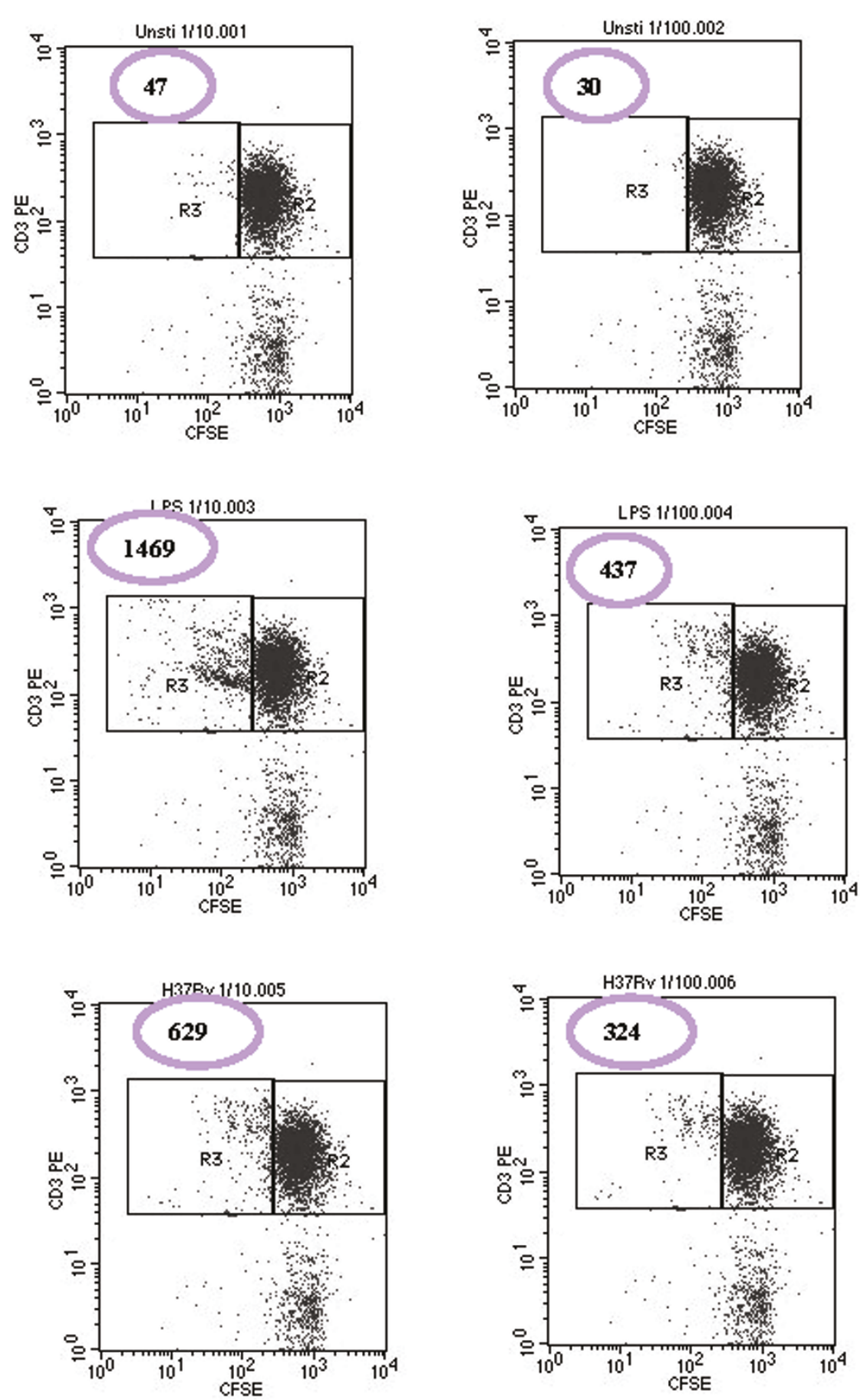

Figure 2 Autologous T cell proliferation at 1:10 and 1:100 of DC: T cell ratio by CFSE dilution technique. The cells were stained with antiCD3 PE and analysed with flow cytometry. In all cases 5000 CD3 ${ }^{+}$CFSE bright events collected (Right Hand Box (R2)); the Number of CD3 ${ }^{+}$ CFSE ${ }^{\text {dim }}$ events (Left Hand Box (R3)) was then determined. The cell division index (CDI) was calculated as described in the methodology.

and production of proinflammatory cytokines that lead to maturation and possibly to migration and effective antigen processing and presentation with a polarization of the immune response towards protective Th-1 type. We have also demonstrated that M. tuberculosis-infected
DCs are capable of inducing $\mathrm{T}$ cell proliferation. In general, DCs could be the basis for the initiation of the immune response leading to protection in the majority of $M$. tuberculosis-infected individuals. However, this aspect requires further investigation. 


\section{Acknowledgements and Funding}

We are very thankful to Dr. Howard Engers and Dr. Abraham Aseffa for their valuable comments during the lab work and during writing of the manuscript. This work was supported by core funds from the Armauer Hansen Research Institute (Addis Ababa, Ethiopia); and Addis Ababa University, School of Graduate Studies (Addis Ababa, Ethiopia).

\section{Author details}

${ }^{1}$ Armauer Hansen Research Institute (AHRI), Addis Ababa, Ethiopia. ${ }^{2}$ Faculty of Medicine, Addis Ababa University, Addis Ababa, Ethiopia. ${ }^{3}$ Faculty of Veterinary Medicine, Addis Ababa, University, Addis Ababa, Ethiopia. ${ }^{4}$ Max Planck Institute for Infection Biology, Berlin, Germany.

\section{Authors' contributions}

AM carried out all the laboratory work, analyzing data and preparing the manuscript. SP contributed in conception, designing the study and correcting the manuscript. GM and MS contributed in laboratory work. AH contributed in quiding laboratory work and correcting the manuscript. All authors read and approved the final manuscript.

\section{Competing interests}

The authors declare that they have no competing interests.

Received: 3 June 2011 Accepted: 21 July 2011 Published: 21 July 2011

\section{References}

1. WHO: Tuberculosis facts. 2007.

2. Lipscomb MF, Masten BJ: Dendritic cells: immune regulators in health and disease. Physiol Rev 2002, 82:97-130.

3. Banchereau J, Briere F, Caux C, Davoust J, Lebecque S, Liu YJ, Pulendran B, Palucka K: Immunobiology of dendritic cells. Annu Rev Immunol 2000, 18:767-811.

4. Henderson RA, Watkins SC, Flynn JL: Activation of human dendritic cells following infection with Mycobacterium tuberculosis. J Immunol 1997, 159:635-643.

5. Hanekom WA, Mendillo M, Manca C, Haslett PA, Siddiqui MR, Barry C, Kaplan G: Mycobacterium tuberculosis inhibits maturation of human monocyte-derived dendritic cells in vitro. J Infect Dis 2003, 188:257-266.

6. Romani N, Gruner S, Brang D, Kampgen E, Lenz A, Trockenbacher B, Konwalinka G, Fritsch PO, Steinman RM, Schuler G: Proliferating dendritic cell progenitors in human blood. J Exp Med 1994, 180:83-93.

7. Mannering SI, Morris J, Jensen K, Purcell A, Honeyman M, van Endert P, Harrison $\mathrm{L}$ : A sensitive method for detecting proliferation of rare autoantigen-specific human T cells. J Immunol Methods 283:173.

8. Fortsch D, Rollinghoff M, Stenger S: IL-10 converts human dendritic cells into macrophage-like cells with increased antibacterial activity against virulent Mycobacterium tuberculosis. J Immunol 2000, 165:978-987.

9. Fontana S, Moratto D, Mangal S, De Francesco M, Vermi W, Ferrari S, Facchetti F, Kutukculer N, Fiorini C, Duse M, Das P, Notarangelo L, Plebani A, Badolato R: Functional defects of dendritic cells in patients with CD40 deficiency. Blood 2003, 12:4099.

10. Kruse M, Rosorius O, Kratzer F, Stelz G, Kuhnt C, Schuler G, Hauber J, Steinkasserer A: Mature dendritic cells infected with herpes simplex virus type 1 exhibit inhibited T-cell stimulatory capacity. J Virol 2000, 74:7127.

11. Fugier-Vivier I, Servet-Delprat C, Rivailler P, Rissoan M, Liu Y, RabourdinCombe C: Measles virus suppresses cell-mediated immunity by interfering with the survival and functions of dendritic and T cells. J Exp Med 1997, 186:813.

12. Kruse M, Rosorius O, Kratzer F, Bevec D, Kuhnt C, Steinkasserer A, Schuler G, Hauber J: Inhibition of CD83 cell surface expression during dendritic cell maturation by interference with nuclear export of CD83 mRNA. J Exp Med 2000, 9:1581.

13. Giacomini E, Sotolongo A, lona E, Severa M, Remoli ME, Gafa V, Lande R, Fattorini L, Smith I, Manganelli R, Coccia EM: Infection of human dendritic cells with a Mycobacterium tuberculosis sigE mutant stimulates production of high levels of interleukin-10 but low levels of CXCL10: impact on the T-cell response. Infect Immun 2006, 74:3296-3304.

14. Xia W, Pinto C, Kradin R: The antigen-presenting activities of la+ dendritic cells shift dynamically from lung to lymph node after an airway challenge with soluble antigen. J Exp Med 1995, 181:1275.
15. Bodnar KA, Serbina NV, Flynn JL: Fate of Mycobacterium tuberculosis within murine dendritic cells. Infect Immun 2001, 69:800-809.

16. Geijtenbeek T, Van Vliet SJ, Koppel EA, Sanchez-Hernandez M, Vandenbroucke-Grauls CM, Appelmelk B, Van Kooyk Y: Mycobacteria target DC-SIGN to suppress dendritic cell function. J Exp Med 2003, 197:7.

doi:10.1186/1756-0500-4-247

Cite this article as: Mihret et al.: Dendritic Cells Activate and Mature after Infection with Mycobacterium tuberculosis. BMC Research Notes 2011 $4: 247$.

\section{Submit your next manuscript to BioMed Central and take full advantage of:}

- Convenient online submission

- Thorough peer review

- No space constraints or color figure charges

- Immediate publication on acceptance

- Inclusion in PubMed, CAS, Scopus and Google Scholar

- Research which is freely available for redistribution 